\title{
Equilibrium uniqueness with perfect complements
}

\author{
Eilon Solan* and Nicolas Vieille ${ }^{\dagger}$
}

May 15, 2003

JEL classification: D50

Keywords: Equilibrium uniqueness, perfect complements, networks.

\section{Introduction}

It is sometimes the case that some economic goods are perfect complements to some agents, but not to all agents. As an illustration, consider the following highly stylized story. A regulator is facing the problem of pricing the use of a given network, e.g., a highway network. For our purposes, a network is simply a graph, with no loop. Each edge of the graph has a capacity: the maximal amount of traffic it can accommodate. Demand for capacity originates from agents, who are considering using their car for commuting between two nodes in the network. Whether an agent is willing to commute with the highway, rather than using some alternative form of transportation, is simply a function of the total price he would have to pay, i.e., of the sum of the prices of the edges he would need to use to reach his destination. An equilibrium price is a vector of prices, one for each edge, such that the capacity of each edge exactly meets the total demand of that edge. The regulator's problem is to set prices at an equilibrium level. Similar issues arise with bandwidth allocation by a monopoly operating a telecommunication network.

Here we prove that the equilibrium allocations are at most unique, under minimal conditions on the demand functions. In addition, we provide somewhat stringent conditions under which equilibrium prices do exist, discuss a few necessary conditions for existence, and a few examples where equilibrium prices fail to exist.

Our contribution relates to three different strands of literature. On the one hand, an important theme in general equilibrium theory has been to

*MEDS Department, Kellogg School of Management, Northwestern University, and School of Mathematical Sciences, Tel Aviv University, Tel Aviv 69978, Israel. e-mail: eilons@post.tau.ac.il, e-solan@kellogg.northwestern.edu

${ }^{\dagger}$ Département Finance et Economie, HEC, 1, rue de la Libération, 78351 Jouy-enJosas, France. e-mail: vieille@hec.fr 
show that, under appropriate substituability conditions between goods, the equilibrium price vector is unique, see, e.g., Pearce and Wise (1973), Iritani (1981), and the references therein. By contrast, we here adopt a partial equilibrium approach, so that the excess demand does not satisfy Walras' law, and the different goods are complements rather than substitutes. Indeed, the various edges of the path used by a given agent are perfect complements, while other edges in the graph are deemed irrelevant by this agent.

On the other hand, within a partial equilibrium framework, it has been argued that (i) assuming away wealth effects, (ii) provided the markets for the different goods are competitive, and (iii) under sufficient regularity on the fundamentals of the economy (cost and utility functions), such an existence and uniqueness result holds, see, e.g., Mas-Colell et al. (1995, Section 10.G). The absence of wealth effects is generally viewed as a reasonable assumption, once agents spend only a small fraction of their income on the set of goods under consideration, see Marshall (1920) and Vives (1987). To contrast with (i-iii), our assumptions on the demand function are of a quite different nature, and the supply side of the economy is not competitive. Indeed, in the above stylized story, the supply curve is vertical. Plainly also, our story puts considerable structure on the demand functions.

Finally, at a broader level, the uniqueness question relates to proving that the excess demand function is globally one-to-one, as a function of the prices of the various edges. Very few sets of sufficient conditions are known, see Gale and Nikaido (1965) and Nikaido (1972). As was verified by Delenda (2002), none of these sufficient conditions applies here.

The note is organized as follows. We first lay down some notation in Section 1, which also contains the existence and uniqueness result. In Section 2 we discuss the existence issue, while Section 3 is devoted to uniqueness.

\section{The model}

It is convenient to deal with a slightly more abstract model than the one sketched in the introduction. We let $X$ be the finite set of goods, and $I$ be the finite set of economic agents. Good $x \in X$ is in fixed supply $Q_{x} \geq 0$.

Each agent $i$ cares only about a subset of goods $X^{i} \subseteq X$, and the different goods in $X^{i}$ are perfect complements for agent $i$. As a consequence, the individual demand of agent $i$, as a function of the price vector $p=\left(p_{x}\right)_{x \in X}$, reduces to a function $D^{i}\left(\sum_{x \in X^{i}} p_{x}\right)$. Throughout the note, we assume that $D^{i}:[0, \infty) \rightarrow[0, \infty]$ is non-increasing, and decreasing whenever positive. ${ }^{1}$ In addition, we assume that $D^{i}(\infty):=\lim _{q \rightarrow \infty} D^{i}(q)=0$, for each $i \in I$, and that $D^{i}(\cdot)$ is finite and continuous, except possibly at 0 , in which case $\lim _{q \rightarrow 0} D^{i}(q)=\infty$. Abusing notations, we sometimes write $D^{i}(p)$ instead of $D^{i}\left(\sum_{x \in X^{i}} p_{x}\right)$.

\footnotetext{
${ }^{1}$ Formally, $D^{i}(q)>D^{i}\left(q^{\prime}\right)$ as soon as $D^{i}(q)>0$ and $q>q^{\prime}$.
} 
The aggregate demand for good $x$ is

$$
\mathcal{D}_{x}(p)=\sum_{i \in I: x \in X^{i}} D^{i}\left(\sum_{y \in X^{i}} p_{y}\right) .
$$

Following classical game theory usage, we denote by $\left(p_{x}, p_{-x}\right)$ the price vector in which the price of good $x$ is $p_{x}$, and the prices of the remaining goods are described by the vector $p_{-x} \in[0, \infty]^{X \backslash\{x\}}$. An equilibrium price is a vector $p \in[0, \infty]^{X}$ such that $\mathcal{D}_{x}(p)=Q_{x}$ for each $x \in X$. The allocation that corresponds to a price vector $p$ is the vector of demands $\left(D^{i}(p)\right)_{i \in I}$. We can now state our result.

Theorem 1 The following holds:

- There is at most one equilibrium allocation.

- If $\mathcal{D}_{x}\left(0_{x}, \infty_{-x}\right)=\sum_{i \in I: X^{i}=\{x\}} D^{i}(0) \geq Q_{x}$ for each $x \in X$, there is an equilibrium price.

We briefly comment on this result. We first observe that the equilibrium price need not be unique. As a trivial illustration, consider the following example.

Example 1: Suppose $X=\{A, B\}, Q_{A}=Q_{B}$, and $X_{i}=\{A, B\}$ for each $i \in I$ : the two goods are perfect complements for all agents. In such a case, the excess demand of each good is a function of the sum of the prices of the two goods, and the set of equilibrium prices is infinite. ${ }^{2}$

Such an example can easily be discarded on the ground that it is here artificial to view the two goods as different marketed goods. However, it is very easy to construct variants of this example in which the fact that all active agents view the two goods as complements is an endogenous characteristic of the market. As an illustration consider the following example.

Here and in the sequel, it is convenient to denote, for every bundle $C \subseteq X$, the demand for bundle $C$ by $D_{C}(\cdot)=\sum_{i \in I: X^{i}=C} D^{i}(\cdot)$. Since the demand functions are decreasing whenever positive, supplying the demand functions for bundles is equivalent to supplying the individual demand functions.

Example 2: $\quad$ Suppose $X=\{A, B\}$, and $Q_{A}=Q_{B}=1$. Suppose that $D_{\{A\}}\left(p_{A}\right)=\left(1 / 2-p_{A}\right) \mathbf{1}_{p_{A}<1 / 2}, D_{\{B\}}\left(p_{B}\right)=\left(1 / 2-p_{B}\right) \mathbf{1}_{p_{B}<1 / 2}$ and $D_{\{A, B\}}\left(p_{A}+p_{B}\right)=\left(5 / 2-\left(p_{A}+p_{B}\right)\right) \mathbf{1}_{p_{A}+p_{B}<5 / 2}$. In this example, the set of equilibrium prices is the set of vectors $\left(p_{A}, p_{B}\right)$, such that $p_{A}+p_{B}=3 / 2$

\footnotetext{
${ }^{2}$ Unless (i) $(0,0)$ is an equilibrium price, in which case it is the unique equilibrium, or (ii) there is no equilibrium price.
} 
and $p_{A}, p_{B} \geq 1 / 2$. Observe that if the common supply $Q_{A}=Q_{B}$ is within the interval $[3 / 2,3]$, the equilibrium price is unique.

The assumption made in the existence statement is unfortunately restrictive. It requires that there is excess demand for good $x$ as soon as the price of good $x$ is low enough, even if the prices of all remaining goods are arbitrarily high. This assumption implies, and is actually equivalent to, the following.

- For each good $x \in X$, there is at least one agent $i$ such that $X^{i}=\{x\}$;

- Moreover, $D_{\{x\}}(0) \geq Q_{x}$.

\section{Equilibrium existence}

Here we discuss the existence issue. We first prove the existence claim in Theorem 1. Next, we discuss possible extensions.

If $Q_{x}=0$ for some good $x$, we set $p_{x}=\infty$, and discard that good. Hence we assume from now on that $Q_{x}>0$ for every good $x \in X$.

Choose $P \in \mathbf{R}^{+}$large enough so that $D^{i}(P)<Q_{x} /|I|$, for each $i \in I$ and $x \in X$. As a consequence of the choice of $P$, one has $\mathcal{D}_{x}(p)<Q_{x}$ for each $x \in$ $X$ and each price vector $p=\left(p_{y}\right)_{y \in X}$ such that $p_{x} \geq P$. Given a price vector $p \in[0, P]^{X}$ and $x \in X$, we let $f_{x}(p) \in[0, P]$ be defined by $\mathcal{D}_{x}\left(f_{x}(p), p_{-x}\right)=$ $Q_{x}$. Thus, $f_{x}(p)$ is a price level for good $x$ that clears the market for good $x$, when all other prices remain unchanged. By the continuity properties of the map $w \mapsto \mathcal{D}_{x}\left(w, p_{-x}\right)$, and since $\mathcal{D}_{x}\left(0, p_{-x}\right) \geq \mathcal{D}_{x}\left(0, \infty_{-x}\right) \geq Q_{x}>$ $\mathcal{D}_{x}\left(P, p_{-x}\right)$, the existence of such a price level is granted. In addition, since $\mathcal{D}_{x}\left(\cdot, p_{-x}\right)$ is decreasing whenever positive, $f_{x}(p)$ is uniquely defined, and is within the range $[0, P]$. Finally, by the continuity properties of the map $\mathcal{D}_{x}$ on $[0, P]^{X}, f_{x}$ is continuous on this range. Therefore, by Brouwer's fixed point Theorem, the function $f:[0, P]^{X} \rightarrow[0, P]^{X}$ defined by $f(p)=$ $\left(f_{x}(p)\right)_{x \in X}$ has a fixed point. Plainly, any fixed point of $f$ is an equilibrium price. This concludes the proof of the existence claim.

The assumption that $\mathcal{D}_{x}\left(0_{x}, \infty_{-x}\right) \geq Q_{x}$ for each $x \in X$ is not quite satisfactory. We investigate below to what extent this assumption can be weakened. Note first that an obvious necessary condition for the existence of an equilibrium price is $\mathcal{D}_{x}(0) \geq Q_{x}$ for each $x \in X$. The discussion proceeds as follows. We first analyze an example in which existence does not hold. Next, we study in detail the two-good model, and thereby illustrate the difficulty of providing a necessary and sufficient condition for the existence of an equilibrium price. We stress that our discussion has no immediate extension to the $n$-good model

Example 3: Assume that $X=\{A, B\}$, with $Q_{A}=Q_{B}=1$, and the demand functions for bundles are given by $D_{\{A\}}\left(p_{A}\right)=\left(5 / 4-p_{A}\right) \mathbf{1}_{p_{A}<5 / 4}$, 
$D_{\{B\}}\left(p_{B}\right)=\left(1 / 4-p_{B}\right) \mathbf{1}_{p_{B}<5 / 4}$, and $D_{\{A, B\}}\left(p_{A}+p_{B}\right)=\left(1-\left(p_{A}+p_{B}\right) / 2\right) \mathbf{1}_{p_{A}+p_{B}<2}$.

We check that no equilibrium price exists. We argue by contradiction, and let $\left(p_{A}^{*}, p_{B}^{*}\right)$ be an equilibrium price. Observe first that $D_{\{A\}}\left(p_{A}^{*}\right)=$ $Q_{A}-D_{\{A, B\}}\left(p_{A}^{*}+p_{B}^{*}\right)=Q_{B}-D_{\{A, B\}}\left(p_{A}^{*}+p_{B}^{*}\right)=D_{\{B\}}\left(p_{B}^{*}\right)$, and note that $(0,0)$ is not an equilibrium price. In particular, $D_{\{A, B\}}\left(p_{A}^{*}, p_{B}^{*}\right)<1$, hence $D_{\{A\}}\left(p_{A}^{*}\right)=D_{\{B\}}\left(p_{B}^{*}\right)>0$. Given the functional form of both $D_{\{A\}}$ and $D_{\{B\}}$, this implies $p_{A}^{*}=p_{B}^{*}+1$. Therefore, the equality $D_{\{B\}}\left(p_{B}^{*}\right)+$ $D_{\{A, B\}}\left(2 p_{B}^{*}+1\right)=Q_{B}=1$ must hold - which is impossible, since $D_{\{B\}}(0)+$ $D_{\{A, B\}}(2 \times 0+1)=1 / 4+1 / 2<1$.

We now provide an exhaustive treatment of the two-good model, and first get rid of somewhat degererate cases. Throughout, we let $X=\{A, B\}$. If $D_{\{A, B\}}(\cdot) \equiv 0$ then the market reduces to two independent markets; indeed, no agent is interested in both goods. Since the demand functions are continuous and decreasing whenever positive, an equilibrium price exists if and only if $D_{\{A\}}(0) \geq Q_{A}$ and $D_{\{B\}}(0) \geq Q_{B}$, and it is then unique.

If $D_{\{A\}}(\cdot) \equiv 0$ - no agent is solely interested in good $A$ - then, if an equilibrium price $\left(p_{A}^{*}, p_{B}^{*}\right)$ exists, it must satisfy $Q_{A}=D_{\{A, B\}}\left(p_{A}^{*}+p_{B}^{*}\right)$, which uniquely defines the sum $p_{A}^{*}+p_{B}^{*}$. Let $c \in \mathbf{R}^{+}$be the unique real number such that $Q_{A}=D_{\{A, B\}}(c)$. Plainly, an equilibrium price exists if and only if $D_{\{B\}}(c)+D_{\{A, B\}}(c) \leq Q_{B} \leq D_{\{B\}}(0)+D_{\{A, B\}}(c)$. Equilibrium prices $\left(p_{A}^{*}, p_{B}^{*}\right)$ are then characterized by (a) $p_{A}^{*}+p_{B}^{*}=c$, and (b) $D_{\{B\}}\left(p_{B}^{*}\right)+D_{\{A, B\}}(c)=Q_{B}$. In particular, the equilibrium price is unique if $D_{\{B\}}\left(p_{B}^{*}\right) \neq 0$.

If $D_{\{B\}}(0) \geq Q_{B}$ and $D_{\{A\}}(0) \geq Q_{A}$, existence of an equilibrium is guaranteed by Theorem 1 .

We are thus left with the case in which none of the functions $D_{\{A\}}(\cdot)$, $D_{\{B\}}(\cdot)$ and $D_{\{A, B\}}(\cdot)$ is identically zero, and, w.l.o.g., $D_{\{B\}}(0)<Q_{B}$. We also assume that $\mathcal{D}_{A}(0)=D_{\{A\}}(0)+D_{\{A, B\}}(0) \geq Q_{A}$ and $\mathcal{D}_{B}(0)=$ $D_{\{B\}}(0)+D_{\{A, B\}}(0) \geq Q_{B}$, since - as said above - these are necessary conditions for equilibrium existence.

For every $q \in \mathbf{R}^{+}$with $D_{\{B\}}(q)+D_{\{A, B\}}(q) \geq Q_{B}$, we let $f(q) \in \mathbf{R}^{+}$ be such that $D_{\{B\}}(q)+D_{\{A, B\}}(q+f(q))=Q_{B}$. The existence of $f(q)$ is granted since the demand functions are continuous and since for every such $q \geq 0$

$D_{\{B\}}(q)+D_{\{A, B\}}(\infty)=D_{\{B\}}(q) \leq D_{\{B\}}(0)<Q_{B} \leq D_{\{B\}}(q)+D_{\{A, B\}}(q)$.

Note also that $f(q)$ is uniquely defined, provided $D_{\{B\}}(q)<Q_{B}$.

For convenience, we set $f(q)=0$ for $q \geq \widehat{q}$, where $\widehat{q} \in \mathbf{R}^{+}$is the unique real number with $D_{\{B\}}(\widehat{q})=Q_{B}$. With this specification, the function $q \mapsto f(q)$ is continuous and strictly decreasing whenever positive.

A pair $\left(p_{A}^{*}, p_{B}^{*}\right)$ in an equilibrium price if and only if (a) $p_{A}^{*}=f\left(p_{B}^{*}\right)$, and (b) $D_{\{A\}}\left(p_{A}^{*}\right)+D_{\{A, B\}}\left(p_{A}^{*}+p_{B}^{*}\right)=Q_{A}$. 
Since the function $q \mapsto D_{\{B\}}(q)$ is non-increasing, the function $q \mapsto$ $D_{\{A, B\}}(q+f(q))=Q_{B}-D_{\{B\}}(q)$ is non-decreasing. Moreover, as the composition of two non-increasing functions, the function $q \mapsto D_{\{A\}}(f(q))$ is non-decreasing. In particular, the function $q \mapsto D_{\{A\}}(f(q))+D_{\{A, B\}}(q+$ $f(q))$ is continuous and non-decreasing.

Therefore, an equilibrium exists if and only if $D_{\{A\}}(f(0))+D_{\{A, B\}}(f(0)) \leq$ $Q_{A} \leq D_{\{A\}}(0)+D_{\{A, B\}}(\widehat{q})$.

\section{Equilibrium uniqueness}

We here prove the uniqueness claim in Theorem 1 . All vectors in the sequel are column-vectors. The proof relies on the following lemma.

Lemma 2 Let $\mathcal{C}$ be a non-empty collection of non-empty subsets of $X$, and let $\pi=\left(\pi_{x}\right)_{x \in X} \in \mathbf{R}^{X}$ be given. Then the system (1) below in the variables $\left(d_{C}\right)_{C \in \mathcal{C}}$ has no solution:

$$
\begin{cases}d_{C} \sum_{x \in C} \pi_{x}>0 & \forall C \in \mathcal{C} \\ \sum_{C \in \mathcal{C}: x \in C} d_{C}=0 & \forall x \in X\end{cases}
$$

Proof. For $C \in \mathcal{C}$ and $x \in X$, define

$$
a_{C, x}= \begin{cases}0 & x \notin C \\ \frac{1}{\sum_{y \in C} \pi_{y}} & x \in C\end{cases}
$$

Denote by $A=\left(a_{C, x}\right)$ the matrix with $|\mathcal{C}|$ rows and $|X|$ columns that consists of all those $a_{C, x}$.

The vector $A \pi$ is given by

$$
(A \pi)_{C}=\sum_{x \in X} a_{C, x} \pi_{x}=\sum_{x \in C} \frac{1}{\sum_{y \in C} \pi_{y}} \pi_{x}=1 .
$$

In particular, the system (S1) $A z \geq 0, A z \neq 0$ has a solution in $\mathbf{R}^{X}$. By Stiemke's Theorem (Stiemke (1915), see also Mangasarian (1969, p. 32)), this implies that the system (S2) $y^{T} A=0, y>0$ has no solution.

To prove that the system (1) has no solution, we prove that any solution of (1) defines a solution of the system (S2).

Let $\left(d_{C}\right)_{C \in \mathcal{C}}$ be a solution of the system (1), and set $y_{C}=d_{C} \sum_{x \in C} \pi_{x}$ for $C \in \mathcal{C}$. By (1), $y_{C}>0$ for every $C \in \mathcal{C}$, and for every $x \in X$,

$$
\left(y^{T} A\right)_{x}=\sum_{C \in \mathcal{C}} y_{C} a_{C, x}=\sum_{C \in \mathcal{C}} d_{C} a_{C, x} \sum_{y \in C} \pi_{y}=\sum_{C \in \mathcal{C}: x \in C} d_{C}=0 .
$$

Hence $y=\left(y_{C}\right)_{C \in \mathcal{C}}$ is a solution to (S2) - a contradiction. 
We now turn to the uniqueness proof. We show that if there are two distinct equilibrium allocations then a solution to the system (1) exists.

Assume that $p$ and $\widehat{p}$ are two different equilibrium price vectors, such that the corresponding equilibrium allocations differ, and set $\pi:=\widehat{p}-p$. As above, for $C \subseteq X$, we set $D_{C}(\cdot):=\sum_{i \in I: X^{i}=C} D^{i}(\cdot)$. We define $\mathcal{C}=\left\{C \subseteq X: D_{C}(\widehat{p}) \neq D_{C}(p)\right\}$ to be the set of bundles for which the demand changes when moving from price $p$ to price $\widehat{p}$. By assumption, the set $\mathcal{C}$ is non-empty. For $C \in \mathcal{C}$, define $d_{C}=D_{C}(p)-D_{C}(\widehat{p}) \neq 0$ to be the change in the demand for the bundle $C$. By the monotonicity assumption on the demand functions, one has $d_{C} \sum_{x \in C} \pi_{x}>0$ for each $C \in \mathcal{C}$. On the other hand, since both $p$ and $\widehat{p}$ are equilibrium prices, for each $x \in X$ one has $\sum_{C \subseteq X: x \in C} D_{C}(p)=Q_{x}=\sum_{C \subseteq X: x \in C} D_{C}(\widehat{p})$. Since $D_{C}(p)=D_{C}(\widehat{p})$ for $C \notin \mathcal{C}$, this yields $\sum_{C \in \mathcal{C}: x \in C} d_{C}=0$. Therefore, $\left(d_{C}\right)_{C \in \mathcal{C}}$ is a solution of the system (1) - a contradiction.

\section{References}

[1] Delenda, A. (2002), mimeo.

[2] Gale, D. and H. Nikaido (1965) The Jacobian matrix and global univalence of mappings, Mathematische Annalen, 159:81-93.

[3] Iritani, J. (1981) On uniqueness of general equilibrium, Review of Economic Studies, 48:167-171.

[4] Mangasarian, O.L. (1969) Nonlinear Programming, McGraw-Hill.

[5] Marshall, A. (1920) Principles of Economics, London, Macmillan.

[6] Mas-Colell, A., Whinston, M.D. and J. Green. (1995) Microeconomic Theory, Oxford University Press.

[7] Nikaido, H. (1972) Convex structures and economic theory, Academic Press.

[8] Pearce, I.F. and J. Wise (1973) On the uniqueness of competitive equilibria, Part I: unbounded demand, Econometrica, 41:817-828.

[9] Stiemke, E. (1915) Uber positive Losungen homogener linearer Gleichungen, Mathematische Annalen, 76:340-342.

[10] Vives, X. (1987) Small income effects: A Marshallian theory of consumer surplus and downward sloping demand, Review of Economic Studies, 54:87-103. 